\title{
Protective effect of catalpol on nicotine-induced injury of alveolar bone and associated underlying mechanisms
}

\author{
YANWU LI $^{1 *}$, XINGAI JIN ${ }^{2 *}$ and LIMIN MAO ${ }^{3}$ \\ Departments of ${ }^{1}$ Periodontology, ${ }^{2}$ Pediatrics, and ${ }^{3}$ Oral and Maxillofacial Surgery, \\ College of Stomatology, Harbin Medical University, Harbin, Heilongjiang 150001, P.R. China
}

Received November 11, 2016; Accepted August 14, 2017

DOI: $10.3892 / \mathrm{mmr} .2017 .7604$

\begin{abstract}
Smoking is an important factor that causes periodontitis, which manifests as alveolar bone injury and absorption, and has a high incidence and unfavorable treatment efficacy. Nicotine causes ischemia and inflammation of the periodontium and inhibits the mineralization of alveolar bones. Previous studies have revealed the anti-tumor biological activities of catalpol, in addition to neuroprotection and anti-inflammation. The present study therefore investigated the underlying protective mechanism of catalpol in alveolar bone injury. A total of $24 \mathrm{Wistar}$ rats were infused with nicotine ( $0.7 \mathrm{mg} / \mathrm{kg}$ for 30 days), followed by subcutaneous injection of catalpol ( $2 \mu \mathrm{g} / \mathrm{kg}$ for 14 days). The loss of alveolar bone was examined, and bone alkaline phosphatase (AP) and osteocalcin levels were examined by ELISA. The expression of tumor necrosis factor (TNF)- $\alpha$ and cyclooxygenase-2 (COX-2) was quantified by reverse transcription-quantitative polymerase chain reaction analysis and western blotting. Treatment with nicotine decreased AP and osteocalcin levels, increased TNF- $\alpha$ and COX-2 expression levels, and led to alveolar bone loss compared with the control group. Catalpol decreased bone loss, increased AP and osteocalcin, and decreased TNF- $\alpha$ and COX-2 expression compared with the nicotine treatment group. Catalpol may alleviate nicotine-induced injury and alveolar bone loss via inhibition of inflammatory factors, and facilitate the mineralization of alveolar bones.
\end{abstract}

\section{Introduction}

Periodontal disease frequently occurs in stomatology and the incidence is rapidly increasing, as it manifests in $>90 \%$ of all

Correspondence to: Dr Limin Mao, Department of Oral and Maxillofacial Surgery, College of Stomatology, Harbin Medical University, 23 Youzheng Street, Harbin, Heilongjiang 150001, P.R. China

E-mail:1iminmao123@163.com

*Contributed equally

Key words: catalpol, nicotine, alveolar bone, tumor necrosis factor- $\alpha$, cyclooxygenase- 2 patients referred to stomatology clinics (1-3). Typical features of periodontal disease are the progressive destruction of tissues, gingival inflammation and loss of supporting tissues (particularly the absorption of alveolar bones), which leads to loosening or detachment of teeth $(4,5)$. A number of factors contribute to the occurrence of periodontal disease. An initiating factor is the existence of pathogens; however, the most important independent risk factor is smoking, although genetics, diabetes and autoimmunity are known factors $(6,7)$. In the clinic, the incidence and severity of smoking-induced periodontal disease are increased compared with those in non-smoking patients, and disease is accompanied by an increased rate of tooth loss (8). Deeper periodontal pockets exist in patients who smoke, and these patients exhibit more severe alveolar bone loss and absorption, which compromises the treatment efficacy (9). Numerous harmful substances exist in tobacco which severely affect public health. As the primary component of tobacco, nicotine causes direct damage to human tissues, and causes indirect injury to humoral/cell immunity via the release of inflammatory mediators $(10,11)$. A study suggested that nicotine may cause ischemia and inflammation in periodontal and gingival tissues, and inhibit the mineralization of alveolar bones (12).

Catalpol is an effective component that is extracted from Radix Rehmanniae (figwort family) in traditional Chinese medicine. It is a benzyl glycoside compound with a small molecular weight $(13,14)$. Studies have demonstrated that catalpol exerts a number of biological activities, including clearing oxygen free radicals, inhibiting the permeability of microvessels, and antioxidant, anti-tumor, anti-fungal/viral, anti-Alzheimer's disease, and anti-inflammatory properties (15). Catalpol may exert its anti-inflammatory effects via multiple routes, including suppression of the overproduction of focal inflammatory mediators, and the protection of tissues by mediating cytokines (16). Therefore, the present study aimed to investigate the protective effect and underlying mechanism of catalpol against nicotine-induced alveolar bone injury. This may aid the clinical treatment and understanding of the pathogenesis of periodontal disease.

\section{Materials and methods}

Animals. A total of 24 healthy male Wistar rats [5 weeks old; specific pathogen free (SPF) grade; body weight, $120 \pm 20 \mathrm{~g}$ ] 
were purchased from the Laboratory Animal Center of Harbin Medical University (Harbin, China). Animals were kept in an SPF facility with fixed temperature $\left(21 \pm 1^{\circ} \mathrm{C}\right)$ and humidity (50-70\%) and a 12/12 h dark-light cycle. All rats were provided with food and water ad libitum.

Rats were used for all experiments, and all procedures were approved by the Animal Ethics Committee of the College of Stomatology, Harbin Medical University (Harbin, China).

Reagents and instruments. Surgical instruments were purchased from Suzhou Sunan Zimmered Medical Instrument Co., Ltd. (Jiangsu, China) (16). Nicotine was purchased from Yipu Ruisi Technology Co., Ltd. (Beijing, China) (16). TRIzol reagent was purchased from Invitrogen (Thermo Fisher Scientific, Inc., Waltham, MA, USA). The bone alkaline phosphatase (AP) assay kit was purchased from Roche Diagnostics (Basel, Switzerland). Polyvinylidene difluoride membranes were from Pall Life Sciences (Port Washington, NY, USA). Chemical reagents for western blotting were purchased from Beyotime Institute of Biotechnology (Haimen, China) (16). Enhanced chemiluminescence reagent was purchased from GE Healthcare (Chicago, IL, USA). Rabbit anti-rat tumor necrosis factor (TNF)- $\alpha$ (cat. no. 3707), cyclooxygenase-2 (COX-2) monoclonal antibodies (cat. no. 12282), rabbit b-actin monoclonal antibody (cat. no. 4907), and goat anti-rabbit horseradish peroxidase conjugated immunoglobulin $\mathrm{G}$ antibody (cat. no. 7074) were purchased from Cell Signaling Technology, Inc. (Danvers, MA, USA). The RNA extraction kit (TRIzol reagent) and high-Capacity cDNA Reverse Transcription kit were purchased from Thermo Fisher Scientific, Inc. Rat-specific osteocalcin assay kit was purchased from BTI Biotechnology Institute UK, Ltd. (Colchester, UK). The microplate reader was produced by BD Biosciences (Franklin Lakes, NJ, USA). The DNA amplifier (Gene Amp PCR system 2400) was purchased from PerkinElmer, Inc. (Waltham, MA, USA). The automatic biochemical analyzer was purchased from Beckman Coulter, Inc. (Brea, CA, USA). Other chemical reagents were purchased from Sangon Biotech Co., Ltd. (Shanghai, China) (10).

Animal grouping. A total of 24 rats were randomly divided into three groups $(n=8)$ : Control group, nicotine group and catalpol group. Nicotine injection was performed to generate the alveolar bone injury model and rat periodontal disease.

Nicotine-induced periodontal disease model and treatment with catalpol. Following 1 week of acclimation, the periodontal disease model was prepared. Prior to surgery, rats were fasted for $12 \mathrm{~h}$. Nicotine $(0.7 \mathrm{mg} / \mathrm{kg})$ in sterile saline was intraperitoneally injected for 30 consecutive days, with an equal volume of saline in the control group (16). In the catalpol treatment group, $2 \mu \mathrm{g} / \mathrm{kg}$ catalpol was subcutaneously injected for 14 days subsequent to generating the nicotine-induced periodontal disease model.

Sample collection. Following model generation, blood samples were collected from rat abdominal aortas. Following incubation for $30 \mathrm{~min}$ at room temperature, blood samples were centrifuged at $1,500 \mathrm{x} \mathrm{g}$ at $4^{\circ} \mathrm{C}$ for $10 \mathrm{~min}$. Supernatants were saved and frozen at $-20^{\circ} \mathrm{C}$. At 1 and 2 weeks during catalpol treatment, four rats from each group were sacrificed.
Periodontal tissue and maxilla samples $(5 \mu \mathrm{m})$ were collected and stored at $-80^{\circ} \mathrm{C}$.

Monitoring of alveolar bone loss level. At 1 and 2 weeks during treatment, the loss of alveolar bone was measured via probing test of first molar on bilateral maxilla as described previously (17).

ELISA analysis. Supernatants were extracted from blood samples to measure the expression profile of bone AP (cat. no. MBS722033, MyBioSource, San Diego, CA, USA) and bone osteocalcin (cat. no. MBS728975, MyBioSource) using ELISA kits, following the manufacturers' protocols. A total of $50 \mu \mathrm{l}$ serially-diluted standard curve samples were added into the wells of a 96-well plate. A total of $50 \mu$ l test samples were added into wells in triplicate. Following incubation, washing buffer was added five times to each well for $30 \mathrm{sec}$. Enzyme label reagent was added into each well $(50 \mu \mathrm{l})$, incubated at $37^{\circ} \mathrm{C}$ for $30 \mathrm{~min}$ and washed. Chromogenic substrates A and B were sequentially added (50 $\mu \mathrm{l}$ each). Following $10 \mathrm{~min}$ development at $37^{\circ} \mathrm{C}$ in the dark, $50 \mu$ l stopping buffer was added to each well to quench the reaction. Optical density values at a wavelength of $450 \mathrm{~nm}$ were measured using a microplate reader within $15 \mathrm{~min}$. Sample concentrations were determined using a linear regression function.

Reverse transcription-quantitative polymerase chain reaction (RT-qPCR) analysis. TRIzol reagent was used to extract the mRNA from periodontal tissues of all rats. cDNA synthesis was performed according to the manufacturer's protocol of the RT kit. Primers were designed based on the sequences of target gene fragments and were synthesized by Yingjun Biotechnology Co., Ltd. (Shanghai, China) $(18,19)$. Primer sequences $\left(5^{\prime}-3^{\prime}\right)$ are presented in Table I.

The qPCR was performed on target genes using the following conditions: $55^{\circ} \mathrm{C}$ for $1 \mathrm{~min}$, followed by 35 cycles at $92^{\circ} \mathrm{C}$ for $30 \mathrm{sec}, 58^{\circ} \mathrm{C}$ for $45 \mathrm{sec}$ and $72^{\circ} \mathrm{C}$ for $35 \mathrm{sec}$. Using GAPDH as the internal reference, fluorescent (SYBR-Green Dye; Thermo Fisher Scientific, Inc.) quantification was performed to obtain cycle threshold values of all standards and samples. Using cycle threshold values of the standards as the reference, linear functions were plotted, on which quantitative analysis was performed by $2^{-\Delta \Delta \mathrm{Cq}}$ method (20).

Western blotting. Total protein was extracted from periodontal tissues. Tissues were homogenized in liquid nitrogen, with the addition of RIPA Lysis and Extraction Buffer (Thermo Fisher Scientific, Inc.) for 15-30 min. Ultrasound was used to rupture cells (4 times for $5 \mathrm{sec}$ ), which were subsequently centrifuged at $10,000 \mathrm{x} \mathrm{g}$ at $4^{\circ} \mathrm{C}$ for $15 \mathrm{~min}$. Supernatants were saved and quantified by Pierce BCA Protein Assay kit (Thermo Fisher Scientific, Inc.). Proteins were separated by SDS-PAGE on a $10 \%$ gel, and were transferred to polyvinylidene difluoride membranes using a semi-dry method. Non-specific binding was blocked using 5\% nonfat milk powder at room temperature for $2 \mathrm{~h}$. Primary antibodies against TNF- $\alpha$ and COX-2 (1:1,000 dilution) were added at $4^{\circ} \mathrm{C}$ overnight. The following day, PBS-Tween-20 was added to wash the membrane, followed by the addition of a goat anti-rabbit secondary antibody (1:2,000 dilution) for $30 \mathrm{~min}$ in the dark. Chromogenic 
Table I. Primer sequences.

\begin{tabular}{lll}
\hline Gene & \multicolumn{1}{c}{ Forward primer, 5'-3' } & Reverse primer, 5'-3' \\
\hline GADPH & ACCAGGTATCTGCTGGTTG & TAACCATGATGTCAGCGTGGT \\
TNF- $\alpha$ & GCATGACCTGCTTATGACTG & TTCGTTCCGCTCAACTCTTA \\
COX & TGCTTATGCATGATGCCGACT & CGCTTCTTCGTCAACTCTTATC
\end{tabular}

TNF- $\alpha$, tumor necrosis factor- $\alpha$; COX-2, cyclooxygenase 2 .

Table II. Probing depth of periodontal tissues (mm).

\begin{tabular}{lcc}
\hline Group & First week $(\mathrm{n}=4)$ & Second week $(\mathrm{n}=4)$ \\
\hline Control & $0.19 \pm 0.03$ & $0.21 \pm 0.02$ \\
Nicotine & $0.87 \pm 0.02^{\mathrm{a}}$ & $1.21 \pm 0.03^{\mathrm{a}}$ \\
Catalpol & $0.79 \pm 0.04^{\mathrm{a}}$ & $0.81 \pm 0.03^{\mathrm{a}, \mathrm{b}}$ \\
\hline
\end{tabular}

${ }^{\mathrm{a}} \mathrm{P}<0.05$ vs. control group; ${ }^{\mathrm{b}} \mathrm{P}<0.05$ vs. nicotine group.

substrate (enhanced chemiluminescence) was added for $1 \mathrm{~min}$ development. The membrane was exposed, scanned and the density of bands was quantified with Quantity One software version 4.6.5 (Bio-Rad Laboratories, Inc., Hercules, CA, USA). Each experiment was repeated four times.

Statistical analysis. SPSS version 16.0 software (SPSS, Inc., Chicago, IL, USA) was used to perform the statistical analysis. Measurement data are expressed as the mean \pm standard deviation. The comparisons among multiple groups were performed using one-way analysis of variance (ANOVA) followed by the least significant difference post hoc test, whereas, two-way ANOVA was performed for comparison of difference between groups at different time points. Linear regression was performed by SPSS software for the quantitative analysis of samples for the ELISA assay based on curve obtained from the standard data. $\mathrm{P}<0.05$ was considered to indicate a statistically significance difference.

\section{Results}

Analysis of probing depth in periodontal tissues. A probing test was performed in periodontal tissues to analyze the injury to alveolar bone induced by nicotine and the protective effect of catalpol. The results revealed a significantly elevated probing depth in the first molar of the bilateral maxilla in the nicotine intervention group compared with the control $(\mathrm{P}<0.05$; Table II). Following catalpol treatment, the probing depth of periodontal tissues became shallower in the first week and exhibited a significant decrease compared with the nicotine group in the second week $(\mathrm{P}<0.05$; Table II).

Loss of rat alveolar bones. The degree of alveolar bone loss was measured by the distance between the top of the alveolar bone ridge and the cemento-enamel junction. The results demonstrated a significantly higher alveolar bone loss in nicotine-treated rats compared with the control group $(\mathrm{P}<0.05$;

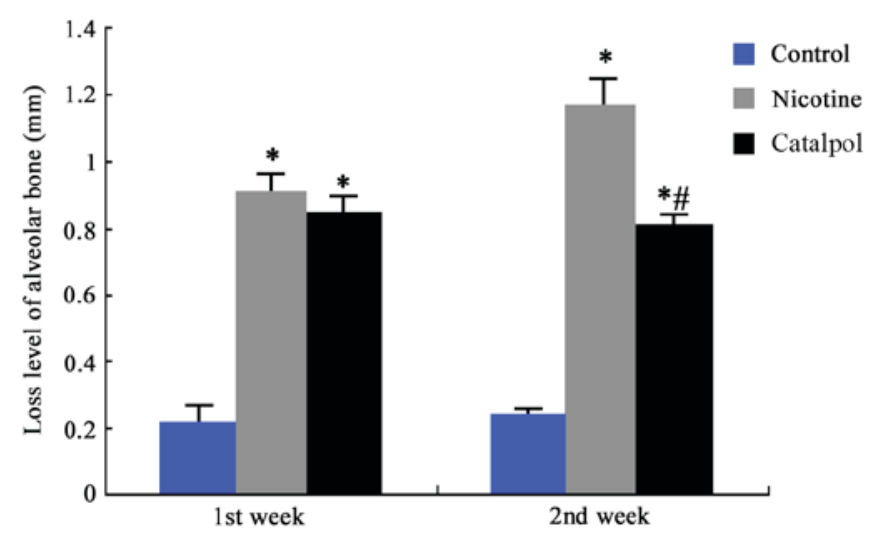

Figure 1. Loss of alveolar bone in nicotine, catalpol or control rats after 1 or 2 weeks. ${ }^{*} \mathrm{P}<0.05$ vs. control group and ${ }^{\#} \mathrm{P}<0.05$ vs. nicotine group.

Fig. 1). Following treatment with catalpol, bone loss improved at the first week; however, this was not significant. At the second week, the bone loss was significantly alleviated compared with the nicotine group $(\mathrm{P}<0.05$; Fig. 1). These results suggested that nicotine may cause significant injury to alveolar bone. Treatment with catalpol, however, may significantly improve and reverse this nicotine-induced injury to alveolar bone.

AP and osteocalcin expression. ELISA analysis was used to detect the bone AP and osteocalcin (OC) level. The results demonstrated significantly decreased AP and OC levels in nicotine-treated rats compared with the control group $(\mathrm{P}<0.05$; Figs. 2 and 3). Following treatment with catalpol, rat AP and OC levels were significantly increased ( $\mathrm{P}<0.05$; Figs. 2 and 3 ).

Expression of TNF- $\alpha$ mRNA in periodontal tissues. The mRNA expression levels of TNF- $\alpha$ in periodontal tissues in rats from all groups was measured. There was significantly elevated TNF- $\alpha$ expression in periodontal tissues in nicotine-treated rats compared with control rats $(\mathrm{P}<0.05$; Fig. 4). Following treatment with catalpol, the TNF- $\alpha$ mRNA expression level was significantly reduced compared with the nicotine group ( $\mathrm{P}<0.05$; Fig. 4).

Expression of COX-2 mRNA in periodontal tissues. The mRNA expression levels of COX-2 in periodontal tissues were measured. There were significantly elevated COX-2 mRNA expression levels in nicotine-treated rat periodontal tissues compared with the control group $(\mathrm{P}<0.05$; Fig. 5). Treatment with catalpol significantly reduced COX-2 mRNA expression levels compared with the nicotine group $(\mathrm{P}<0.05$; Fig. 5). 


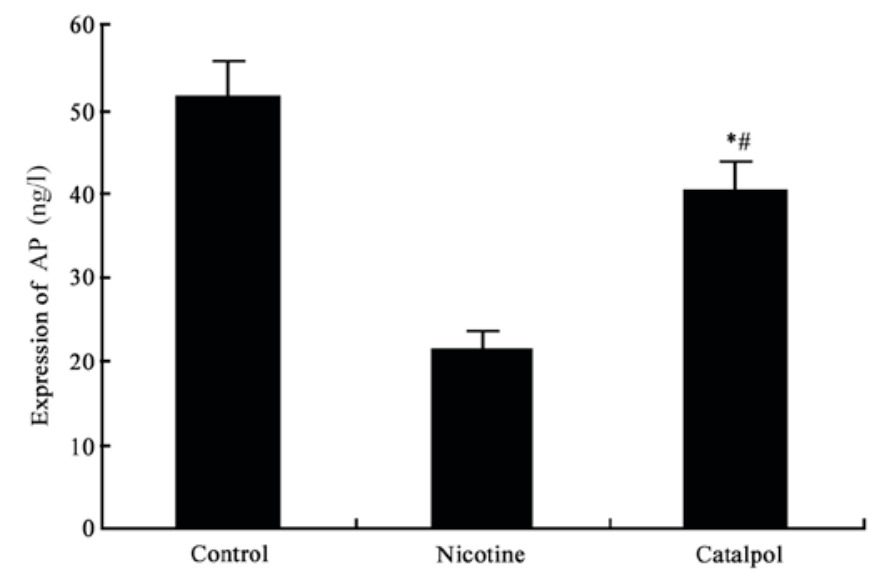

Figure 2. Blood concentration of AP in nicotine, catalpol or control rats. ${ }^{*} \mathrm{P}<0.05$ vs. control group and ${ }^{\#} \mathrm{P}<0.05$ vs. nicotine group. AP, alkaline phosphatase.

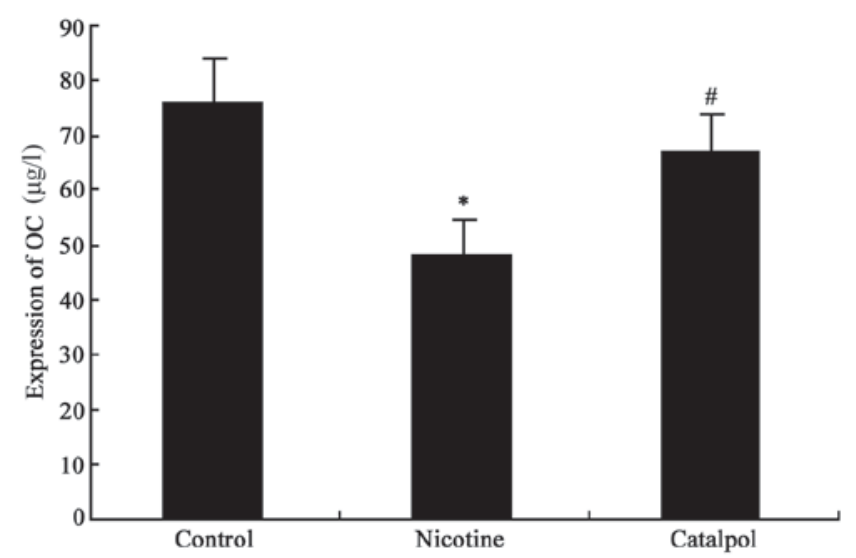

Figure 3. Concentration of $\mathrm{OC}$ in the bones of nicotine, catalpol or control rats. ${ }^{*} \mathrm{P}<0.05$ vs. control group and ${ }^{\#} \mathrm{P}<0.05$ vs. nicotine group. $\mathrm{OC}$, osteocalcin.

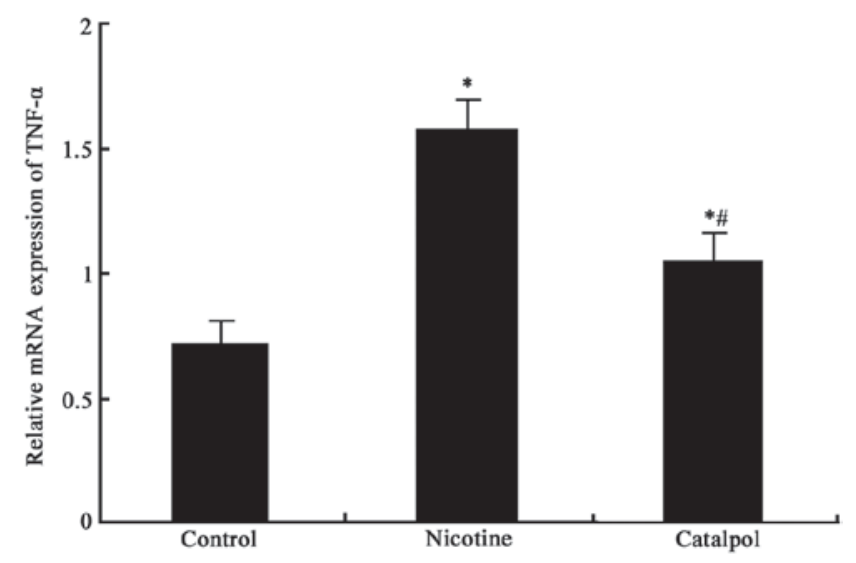

Figure 4. TNF- $\alpha$ mRNA expression levels in periodontal tissues of nicotine, catalpol or control rats. ${ }^{*} \mathrm{P}<0.05$ vs. control group and ${ }^{\#} \mathrm{P}<0.05$ vs. nicotine group. TNF- $\alpha$, tumor necrosis factor- $\alpha$.

$T N F-\alpha$ and COX-2 protein expression. Western blotting was further employed to analyze the expression levels of TNF- $\alpha$ and COX-2 proteins in rat periodontal tissues. Results revealed significantly elevated protein levels of TNF- $\alpha$ and COX-2 after

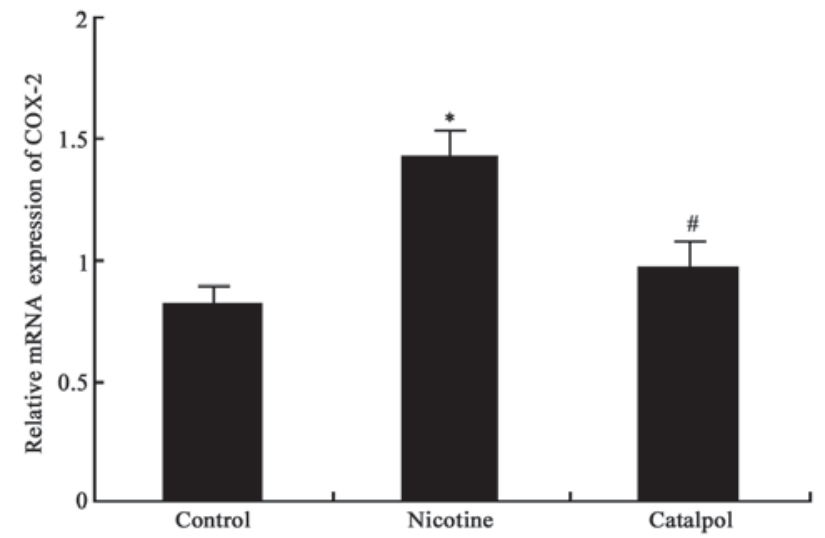

Figure 5. COX-2 mRNA expression levels in periodontal tissues of nicotine, catalpol or control rats. ${ }^{*} \mathrm{P}<0.05$ vs. control group and ${ }^{\text {}} \mathrm{P}<0.05$ vs. nicotine group. COX-2, cyclooxygenase- 2 .

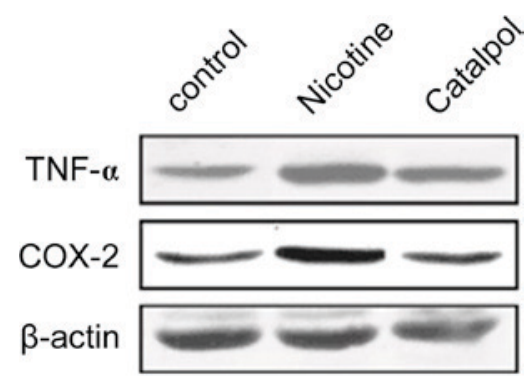

Figure 6. TNF- $\alpha$ and COX-2 protein expression levels in periodontal tissues of nicotine, catalpol or control rats. TNF- $\alpha$, tumor necrosis factor- $\alpha$; COX-2, cyclooxygenase- 2 .

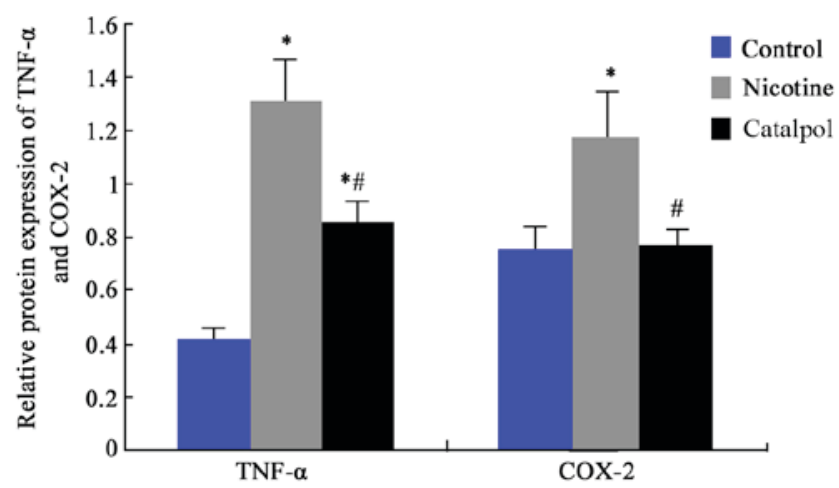

Figure 7. Densitometric analysis of TNF- $\alpha$ and COX-2 protein expression levels in periodontal tissues of nicotine, catalpol or control rats. ${ }^{*} \mathrm{P}<0.05$ vs. control group and ${ }^{~} \mathrm{P}<0.05$ vs. nicotine group. TNF- $\alpha$, tumor necrosis factor- $\alpha$; COX-2, cyclooxygenase- 2 .

nicotine treatment compared with the control group $(\mathrm{P}<0.05$; Figs. 6 and 7). Treatment with catalpol significantly decreased the protein levels in periodontal tissues compared with the nicotine group $(\mathrm{P}<0.05$; Figs. 6 and 7$)$.

\section{Discussion}

Smoking is a risk factor for periodontal disease as it may elevate the presence of pathogens in periodontal tissues, and increase the incidence of hemorrhage. Excessive smoking for 
long periods of time leads to destruction of periodontal tissues. Periodontal disease in smokers is often associated with severe alveolar injury (10). As an important component of tobacco, nicotine may cause body tissue damage accompanied by an inflammatory response. It may induce severe injury to alveolar bones via inhibition of mineralization $(19,21)$. The present study used a periodontal disease model and induced alveolar bone injury via nicotine infusion, and demonstrated that nicotine caused the loss of alveolar bone. Extracted from a traditional Chinese medicine, catalpol exhibits multiple pharmaceutical activities and serves important roles as an anti-inflammatory agent and mediator of oxidation-reduction homeostasis (22). The present study demonstrated that catalpol may alleviate the loss of alveolar bone in a nicotine-induced rat alveolar bone injury model.

AP primarily exists in liver, kidney and bone tissues. It is an extracellular enzyme secreted by osteoblasts, and may additionally be produced from periodontal membrane cells and alveolar bone cells. It may affect the synthesis of osteoblasts during maturation, and is an important marker for bone mineralization and activity of osteoblasts (23). Osteocalcin is secreted by osteoblasts during the matrix mineralization stage, and primarily facilitates bone mineralization (24). The present study demonstrated that nicotine inhibited AP and osteocalcin expression, thereby leading to injury to alveolar bone. Following treatment with catalpol, the secretion levels of AP and osteocalcin were somewhat restored, suggesting that catalpol may exert a protective effect on alveolar bone.

Following entry into the body, nicotine directly initiates the secretion and release of large quantities of inflammatory factors into the serum. This leads to an imbalance of oxygen free radicals in periodontal tissues, production of inflammatory mediators including TNF- $\alpha$ and prostaglandin, periodontal inflammation, destruction of periodontal tissues and injury to alveolar bone (25). COX-2 is an important enzyme that catalyzes the synthesis of prostaglandin from arachidonic acid. The present study, therefore, analyzed the effect of nicotine on the expression of TNF- $\alpha$ and COX-2 in periodontal tissues. The results revealed that nicotine may facilitate the mRNA and protein expression of TNF- $\alpha$ and COX- 2 in periodontal tissues, which may aggravate inflammation and cause further damage to alveolar bone. Further treatment using catalpol, which has notable anti-inflammatory and antioxidant effects, inhibited the expression of TNF- $\alpha$ and COX-2 (at the mRNA and protein levels). This suggested that catalpol may alleviate tissue injury to the periodontium, protect alveolar bone and slow the progression of periodontal disease via inhibition of cytokines and mediation of the homeostasis of oxygen free radicals $(26,27)$.

In conclusion, catalpol may facilitate the mineralization of alveolar bone via inhibition of inflammatory factors, and thus improve nicotine-induced injury to alveolar bone. The present study may provide a reference to the pathogenesis of periodontal disease, and may increase treatment choices for alveolar bone injury.

\section{Acknowledgements}

The authors would like to thank Dr Su Ma and Dr Peihong Liu (Harbin Medical University) for their support and assistance with this manuscript.

\section{References}

1. Trubiani O, Giacoppo S, Ballerini P, Diomede F, Piattelli A, Bramanti P and Mazzon E: Alternative source of stem cells derived from human periodontal ligament: A new treatment for experimental autoimmune encephalomyelitis. Stem Cell Res Ther 7: 1, 2016.

2. Toregeani JF,NassarCA,NassarPO,Toregeani KM,GonzattoGK, Vendrame R, Castilhos JS, Rotta LS, Reinheimer AC, Longoni A and Barcella MW: Evaluation of periodontitis treatment effects on carotid intima-media thickness and expression of laboratory markers related to atherosclerosis. Gen Dent 64: 55-62, 2016.

3. Yang J, Zhang Q, Chen M, Wu WZ, Wang R, Liu CJ, Li B, Shi XL, Du HS and Tan HB: Association between Helicobacter pylori infection and risk of periodontal diseases in Han Chinese: A case-control study. Med Sci Monit 22: 121-126, 2016.

4. Pullishery F, Panchmal GS and Siddique S: Salivary thiocyanate, uric acid and $\mathrm{pH}$ as biomarkers of periodontal disease in tobacco users and non-users- an in-vitro study. J Clin Diagn Res 9: ZC47-ZC50, 2015.

5. Wyganowska-Swiatkowska M and Nohawica MM: Effect of tobacco smoking on human gingival and periodontal fibroblasts. A systematic review of literature. Przegl Lek 72: 158-160, 2015.

6. Warad S, Kalburgi NB, Manak M, Kalburgi VC, Koregol AC, Patanashetti J, Rao S and Kokatnur MV: Determining the effect of Gutkha on serum levels of vitamin B12 and folic acid as compared to smoking among chronic periodontitis subjects: A cross-sectional study. J Clin Diagn Res 8: ZC85-ZC89, 2014.

7. Ng TK, Huang L, Cao D, Yip YW, Tsang WM, Yam GH, Pang CP and Cheung HS: Cigarette smoking hinders human periodontal ligament-derived stem cell proliferation, migration and differentiation potentials. Sci Rep 5: 7828, 2015.

8. Archana MS, Bagewadi A and Keluskar V: Assessment and comparison of phagocytic function and viability of polymorphonuclear leukocytes in saliva of smokers and non-smokers. Arch Oral Biol 60: 229-233, 2015.

9. Huang R, Li M, Ye M, Yang K, Xu X and Gregory RL: Effects of Nicotine on Streptococcus gordonii growth, biofilm formation, and cell aggregation. Appl Environ Microbiol 80: 7212-7218, 2014.

10. Lallier TE, Maturin E, Brady M, Stoute D and Ward T: Resistance to cigarette smoke is increased in periodontal ligament cells by attachment to collagen and fibronectin. J Periodontol 86: 91-100, 2015.

11. Imamura K, Kokubu E, Kita D, Ota K, Ishihara $\mathrm{K}$ and Saito A: Cigarette smoke condensate modulates migration of human gingival epithelial cells and their interactions with Porphyromonas gingivalis. J Periodontal Res 50: 411-421, 2015.

12. Hedna VS, Ansari S, Shahjouei S, Cai PY, Ahmad AS, Mocco J and Qureshi AI: Validity of laser doppler flowmetry in predicting outcome in murine intraluminal middle cerebral artery occlusion stroke. J Vasc Interv Neurol 8: 74-82, 2015.

13. Xue B, Ma B, Zhang Q, Li X, Zhu J, Liu M, Wu X, Wang C and Wu Z: Pharmacokinetics and tissue distribution of Aucubin, Ajugol and Catalpol in rats using a validated simultaneous LC-ESI-MS/MS assay. J Chromatogr B Analyt Technol Biomed Life Sci 1002: 245-253, 2015.

14. Wang JM, Yang LH, Zhang YY, Niu CL, Cui Y, Feng WS and Wang GF: BDNF and COX-2 participate in anti-depressive mechanisms of catalpol in rats undergoing chronic unpredictable mild stress. Physiol Behav 151: 360-368, 2015.

15. Xu Z, Zhang L, Li X, Jiang Z, Sun L, Zhao G, Zhou G, Zhang H, Shang $J$ and Wang T: Mitochondrial fusion/fission process involved in the improvement of catalpol on high glucose-induced hepatic mitochondrial dysfunction. Acta Biochim Biophys Sin (Shanghai) 47: 730-740, 2015.

16. Tewari D, Khan MP, Sagar N, China SP, Singh AK, Kheruka SC, Barai S, Tewari MC, Nagar GK, Vishwakarma AL, et al: Ovariectomized rats with established Osteopenia have Diminished Mesenchymal stem cells in the bone marrow and Impaired Homing, Osteoinduction and Bone Regeneration at the fracture site. Stem Cell Rev 11: 309-321, 2015.

17. Souza DM, Ricardo LH, Kantoski KZ and Rocha RF: Influence of alcohol consumption on alveolar bone level associated with ligature-induced periodontitis in rats. Braz Oral Res 23: 326-332, 2009.

18. Javed F, Bashir Ahmed H and Romanos GE: Association between environmental tobacco smoke and periodontal disease: a systematic review. Environ Res 133: 117-122, 2014. 
19. Tao JH, Zhao M, Wang DG, Yang C, Du LY, Qiu WQ and Jiang S: Biotransformation and metabolic profile of catalpol with human intestinal microflora by ultra-performance liquid chromatography coupled with quadrupole time-of-flight mass spectrometry. J Chromatogr B Analyt Technol Biomed Life Sci: 1009-1010: 163-169, 2016.

20. Livak KJ and Schmittgen TD: Analysis of relative gene expression data using real-time quantitative PCR and the 2(-Delta Delta C(T)) method. Methods 25: 402-408, 2001.

21. Fernandes BS, Hodge JM, Pasco JA, Berk M and Williams LJ: Effects of depression and serotonergic antidepressants on bone: Mechanisms and implications for the treatment of depression. Drugs Aging 33: 21-25, 2016.

22. Fernandes G, Wang C, Yuan X, Liu Z, Dziak R and Yang S: Combination of controlled release platelet-rich plasma alginate beads and bone morphogenetic Protein-2 genetically modified mesenchymal stem cells for bone regeneration. J Periodontol 87: 470-480, 2016

23. Gołąek K, Ostrowska Z, Ziora K, Oświęcimska J, Świętochowska E, Marek B, Kajdaniuk D, Strzelczyk J and Kos-Kudła B: Association between omentin-1, bone metabolism markers, and cytokines of the RANKL/RANK/OPG system in girls with anorexia nervosa. Endokrynol Pol 66: 514-520, 2015.
24. Wadhwa D, Bey A, Hasija M, Moin S, Kumar A, Aman S and Sharma VK: Determination of levels of nitric oxide in smoker and nonsmoker patients with chronic periodontitis. J Periodontal Implant Sci 43: 215-220, 2013.

25. Lazăr L, Loghin A, Bud ES, Cerghizan D, Horváth E and Nagy EE: Cyclooxygenase-2 and matrix metalloproteinase-9 expressions correlate with tissue inflammation degree in periodontal disease. Rom J Morphol Embryol 56: 1441-1446, 2015.

26. Liu JY, Zheng CZ, Hao XP, Zhang DJ, Mao AW and Yuan P: Catalpol ameliorates diabetic atherosclerosis in diabetic rabbits. Am J Transl Res 8: 4278-4288, 2016.

27. Tian YY, An LJ, Jiang L, Duan YL, Chen J and Jiang B: Catalpol protects dopaminergic neurons from LPS-induced neurotoxicity in mesencephalic neuron-glia cultures. Life Sci 80: 193-199, 2006. 